\title{
Comportamiento de voto y nivel de disenso de España en el Consejo de la Unión Europea (2009-2019)
}

\author{
Javier Arregui \\ Universitat Pompeu Fabra \\ javier.arregui@upf.edu \\ ORCID: 0000-0002-1149-7150 \\ Guillermo Bezzina \\ Barcelona Center for European Studies (BACES) \\ guillermo.bezzina@gmail.com
}

Cita recomendada: ARREGUI, J. \& BEZZINA, G. (2022). “Comportamiento de voto y nivel de disenso en España en el Consejo de la Unión Europea (2009-2019)”. Quaderns IEE: Revista de l'Institut d'Estudis Europeus, 1 (1), 4-26. DOI « https://doi.org/10.5565/rev/10.5565/rev/quadernsiee.17 »

\section{Resumen}

El presente artículo analiza el comportamiento de voto de España y de otros Estados miembros en el Consejo de la UE. La investigación se centra en examinar el nivel de disenso que existe en el voto que los Estados miembros emiten al final del proceso de toma de decisiones. Al mismo tiempo, explora una serie de variables que nos pueden ayudar a entender los principales mecanismos explicativos que existen detrás del comportamiento de voto de los Estados miembros de la UE. El análisis se centra, en particular, en el caso de España y en qué mecanismos hay detrás de su comportamiento de voto. Para poder llevar a cabo este estudio hemos elaborado una base de datos que recoge todas las votaciones que ha habido en el Consejo de Ministros en las dos últimas legislaturas: 2009-2014 y 2014-2019. El análisis sistemático de los datos muestra que el disenso sigue siendo un fenómeno muy raro en el Consejo de la UE, con la excepción del Reino Unido en los años anteriores al Brexit. España muestra un nivel de disenso medio con relación al resto de Estados miembros. Los factores que mejor explican el comportamiento de voto tienen que ver con las interrelaciones económicas entre estados, el tamaño del país, las preferencias de los Estados miembros en las diversas áreas temáticas y, en menor medida, la ideología de los gobiernos de los Estados miembros.

Palabras clave: Consejo de la UE; Disenso; Proceso de toma de decisiones; España. 
Abstract. Voting behavior and level of dissent of Spain in the Council of the European Union (2009-2019)

This article analyzes the voting behavior of Spain and other Member States in the Council of the EU. The research focuses on examining the level of dissent that exists in the vote that Member states cast at the end of the decision-making process. At the same time, it explores a series of variables that can help us understand the main explanatory mechanisms that exist behind the voting behavior of the EU Member States. The analysis focuses, in particular, on the case of Spain and on what mechanisms are behind its voting behavior. In order to carry out this study, we have developed a database that collects all the votes that have taken place in the Council of Ministers in the last two legislatures: 2009-2014 and 2014-2019. Systematic analysis of the data shows that dissent remains a very rare phenomenon in the Council of the EU, with the exception of the UK, in the years before Brexit. Spain shows a medium level of dissent in relation to the rest of the Member states. The factors that best explain voting behavior have to do with the economic interrelationships between states, the size of the country, the preferences of the Member states in the various thematic areas and, to a lesser extent, the ideology of the governments of the Member states.

Keywords: Council of the EU; Dissent; Decision-making process; Spain.

Resum. Comportament de vot i nivell de dissens d'Espanya al Consell de la Unió Europea (2009-2019)

Aquest article analitza el comportament de vot d'Espanya i d'altres Estats membres al Consell de la UE. La investigació se centra a examinar el nivell de dissens que hi ha en el vot que els Estats membres emeten al final del procés de presa de decisions. Alhora, explora una sèrie de variables que ens poden ajudar a entendre els principals mecanismes explicatius que hi ha darrere del comportament de vot dels Estats membres de la UE. L'anàlisi se centra, en particular, en el cas d'Espanya i quins mecanismes hi ha darrere del seu comportament de vot. Per poder dur a terme aquest estudi, hem elaborat una base de dades que recull totes les votacions que hi ha hagut al Consell de Ministres en les dues darreres legislatures: 2009-2014 i 2014-2019. L'anàlisi sistemàtica de les dades mostra que el dissens continua sent un fenomen molt rar al Consell de la UE, amb l'excepció del Regne Unit els anys anteriors al Brexit. Espanya mostra un nivell de dissens mitjà en relació amb la resta d'Estats membres. Els factors que millor expliquen el comportament de vot tenen a veure amb les interrelacions econòmiques entre estats, la mida del país, les preferències dels Estats membres a les diverses àrees temàtiques $i$, en menor mesura, la ideologia dels governs dels Estats membres .

Paraules clau: Consell de la UE; Dissentiment; Procés de presa de decisions; Espanya. 


\author{
Sumario \\ 1. Introducción \\ 2. Revelando el comportamiento del voto de los Estados miembros en el Consejo \\ 3. Diseño de la investigación y metodología \\ 4. Análisis descriptivo \\ 5. Análisis: correlaciones y gráficos de dispersión \\ 6. Conclusión \\ Financiación \\ Referencias
}

\title{
1. INTRODUCCIÓN
}

El presente artículo analiza de un modo sistemático el comportamiento del voto y el nivel de disenso de los Estados miembros del Consejo de la Unión Europea (o Consejo de Ministros de la Unión Europea). Después de la negociación de cada dosier legislativo en el Consejo de Ministros, los Estados miembros tiene la oportunidad de emitir un voto final. El estadio del voto del Consejo de Ministros es el estadio final y el momento prominente del proceso de decisión. En este sentido, poder analizar el comportamiento de voto de los Estados miembros proporciona una oportunidad única para refinar nuestro entendimiento sobre el funcionamiento del proceso de toma de decisiones de la UE. La literatura ha dedicado hasta la fecha una atención muy limitada a la hora de identificar y explicar los patrones de voto de los Estados miembros dentro del Consejo de la UE, sobre todo en los últimos años. Es por ello que este artículo plantea las siguientes preguntar de investigación: ¿Cuál es el nivel de disenso de España y de otros Estados miembros en el voto que emiten en el proceso de toma de decisiones? ¿Qué factores pueden ayudar a identificar explicaciones del consenso y/o disenso que tiene lugar dentro del Consejo de Ministros de la UE?. Estas preguntas son relevantes en la medida en que la Unión Europea (UE, en adelante) no adopta ninguna decisión sin la participación y la aprobación del Consejo de Ministros. Esto se refiere a la legislación secundaria: decisiones, reglamentos y directivas, que son los tres tipos de normas que forman el núcleo de la legislación que se elabora en Bruselas.

El proceso decisorio dentro del Consejo de Ministros de la UE viene precedido por una intensa labor del Comité de Representantes Permanentes (Coreper). Al final de este proceso, los comités y grupos de trabajo del Coreper formulan las deliberaciones que han tenido lugar como Puntos A o Puntos B. Los Puntos A ya han sido decididos por los comités del Coreper y, por tanto, ya se ha llegado a un acuerdo y no se discuten en el Consejo de Ministros, mientras que en los Puntos B no ha habido 
acuerdo, y por tanto, se ha de adoptar una decisión y un voto final sobre esos temas. Claramente, estos son los temas con los mayores niveles de controversia dentro de cada dosier legislativo. Después del Tratado de Lisboa, una mayoría de decisiones se adoptan por mayoría cualificada.

El proceso de toma de decisiones en el Consejo de Ministros de la UE tiene lugar de un modo poco inteligible para el ciudadano medio y con un nivel de transparencia y de rendimiento de cuentas muy limitado (Arregui 2012: 97). Por ejemplo, las actas del Consejo por las que se adoptan las decisiones no se hacen públicas en la mayoría de las ocasiones (Arregui 2012). Posiblemente, esta es la principal razón por la que existen pocos estudios sistemáticos sobre el comportamiento de decisión de los Estados miembros dentro del Consejo de Ministros de la UE.

Los estudios que existen en la literatura. hasta la fecha, se focalizan en periodos de tiempo que no van más allá de la década del 2000, en el momento anterior y posterior a la gran ampliación del año 2004. En este sentido, los estudios de Mattila 2004, 2008, 2009; Hayes-Renshaw et al. 2006; Hagemann 2008; Hosli et al., 2011; and Plechanová 2011 se focalizan en los patrones de comportamiento del voto de los Estados miembros en esos años, por ejemplo, identificando qué países votaron de un modo similar. Después de esos años, es decir, en la última década, no se han elaborado estudios que muestren la evolución del comportamiento del voto dentro del Consejo de la UE.

Este artículo va a complementar esas investigaciones previas con tres tipos de innovaciones. En primer lugar, abarca un periodo temporal más reciente, en particular, se focaliza en las dos últimas legislaturas de la UE (2009-2014 y 2014-2019), una vez que la ampliación de los países de Europa del Este está ya plenamente consolidada y sobre las que apenas existen estudios en la literatura. Además, esos fueron años determinantes para la evolución reciente de la UE y su futuro más inmediato, debido a las crisis económica, financiera y política sufrida en los recientes años. Por tanto, los datos disponibles abarcan un amplio periodo temporal de 10 años que permiten extraer conclusiones y generalizaciones, ya que no se ciñen solo a breves periodos temporales que no permitirían obtener una imagen clara y global del comportamiento del voto. En segundo lugar, desagrega los datos de un modo más elaborado que estudios previos, por ejemplo, mostrando información sistemática y precisa sobre los patrones de voto en las diferentes formaciones del Consejo y áreas políticas, en diferentes procedimientos legislativos e, incluso, por tipo de ley (reglamentos, directivas y decisiones). Esto posibilitará profundizar en el análisis comparado entre Estados miembros. En tercer lugar, la presente investigación focaliza su análisis en el caso de España, en cómo vota con relación a otros Estados miembros, e intenta identificar posibles variables que expliquen el sentido de voto que España lleva a cabo dentro del Consejo de la UE. Esto es inédito en la literatura, ya que ningún estudio anterior se había focalizado en el comportamiento de voto de España en perspectiva comparada. 
La siguiente sección elabora algunas de las variables explicativas con las que la literatura ha propuesto explicaciones sobre el voto de los Estados miembros dentro del Consejo. La tercera sección desarrolla el diseño de investigación, prestando especial atención a la elaboración de la base de datos con las que se ha llevado a cabo el análisis. Las secciones subsiguientes presentan el análisis de los datos en sus diferentes versiones, tanto en los análisis de naturaleza descriptiva como de naturaleza más explicativa. En la última sección, se reúnen los principales hallazgos de la investigación y se contextualizan estos hallazgos, aportando explicaciones y conclusiones sobre las preguntas de investigación planteadas.

\section{REVELANDO EL COMPORTAMIENTO DEL VOTO DE LOS ESTADOS MIEMBROS EN EL CONSEJO}

El primer indicador que la literatura ha aportado a la hora de explicar el comportamiento de voto de los Estados miembros en el Consejo de Ministros son las preferencias que cada Estado miembro defiende en el proceso de negociación. Dada la diversidad de intereses políticos y económicos que existen entre los Estados miembros de la UE, estos tienden a tener y a mostrar preferencias diferentes a lo largo del proceso de negociación. De este modo, los Estados miembros manifiestan (con bastante frecuencia) preferencias desiguales que son consecuencia directa de la heterogeneidad de los factores políticos, ideológicos, económicos, institucionales o estratégicos que habitan en los Estados miembros (ver Arregui 2015). Además, la literatura también ha mostrado que la intensidad de las preferencias que los Estados miembros utilizan a la hora de defender un tema político puede ser tan determinante o más que las preferencias que tienen los Estados a la hora de definir el sentido de su voto. Por ejemplo, Arregui y Creighton (2018) muestran que la intensidad de las preferencias en los temas medioambientales son determinantes a la hora de activar el voto en una dirección o en otra. Estos factores han sido ampliamente reconocidos en la literatura especializada sobre el proceso de toma de decisiones dentro del Consejo de Ministros (ver Bueno de Mesquita 1994; Achen 2006; Hoyland and Hansen 2014).

Esta investigación plantea una aproximación a este tipo de factores que proporcionan información sobre la lógica con la que los Estados miembros votan en el Consejo. A priori podemos pensar que los factores de tipo político y económico son los que más peso pueden tener. Esto es así, en la medida en que en los últimos años la UE ha encadenado una serie de crisis económicas y políticas importantes, y, por tanto, es previsible que los condicionamientos de naturaleza económica y/o política hayan podido desempeñar un papel relevante en el proceso decisorio del Consejo de Ministros. Por otro lado, existen otros factores, que están recogidos en la literatura, y que puede ser importante verificar, tales como factores de naturaleza ideológica o institucional de los Estados miembros (Mattila 2004; 2009) o los que están directamente relacionados con la propia naturaleza de los Estados miembros, tales como el tamaño del Estado miembro (y por tanto, la amplitud de temas relevante que 
cubre su comportamiento en el Consejo de Ministros) o la interdependencia e interrelación económica entre los Estados miembros a la hora de poder explicar posibles patrones de cooperación entre Estados miembros y que, por tanto, pueda existir como condicionante del voto (Moravcsik 1998).

\section{DISEÑO DE LA INVESTIGACIÓN Y METODOLOGÍA}

Este análisis está realizado a partir de la información publicada por el portal VoteWatch Europe ${ }^{1}$. Los datos analizados recogen información sobre diferentes votaciones comprendidas en un periodo de tiempo que va desde el año 2009 hasta 2019, es decir, las legislaturas 7 y 8 del Parlamento Europeo. La información que el Consejo hace pública es limitada y solo se refiere a las votaciones finales realizadas a nivel ministerial en propuestas de carácter legislativo o presupuestario. VoteWatch Europe recoge esa información, a partir de la página web del Consejo ${ }^{2}$ y de EUR-Lex 3 . A partir de las diferentes informaciones recogidas por esta fuente, hemos procedido a realizar una base de datos que incluye diferentes variables de interés, tales como la configuración del Consejo, el área política, el tipo de procedimiento legislativo, el tipo de ley o la regla de votación. Todo ello nos dará conocimiento de primera mano sobre cómo se ha votado en el Consejo de Ministros de la UE durante las dos últimas legislaturas.

El dataset está compuesto por un total de 32.784 observaciones que corresponden a 1.185 votaciones. Es decir, para cada votación se obtiene una observación para cada Estado Miembro (28, desde la incorporación de Croacia el 1 de Julio de 2013). La principal ventaja de este dataset es la elevada cantidad de observaciones que contiene, convirtiéndose en el más completo realizado hasta la fecha, tanto por la cantidad de información como el amplio periodo que abarca, recogiendo los datos de manera uniforme y sistematizada. Esta base de datos se focaliza, por tanto, en el comportamiento de voto de los Estados miembros, más allá de las preferencias que hayan manifestado durante el proceso de negociación política.

Como ya se ha indicado, un objetivo del análisis es describir el comportamiento de voto de los Estados miembros, especialmente de España, en el Consejo de la UE. Por tanto, la metodología usada en este artículo para explotar esta base de datos es, básicamente, de naturaleza comparada. Para ello, se utiliza una metodología descriptiva y técnicas de análisis bivariados. A través de la descripción en detalle de los

\footnotetext{
${ }^{1}$ El sitio web VoteWatch Europe (https://www.votewatch.eu/) cubre toda la actividad de votación en el Parlamento Europeo y el Consejo de Ministros desde julio de 2009. Además, proporciona estadísticas para el Parlamento Europeo que se remontan a julio de 2004.

2 https://www.votewatch.eu/en/term9-council-latest-votes.html

${ }^{3}$ El portal EUR-Lex (https://eur-lex.europa.eu/) permite conocer el ciclo de vida de una propuesta de legislación, desde el momento en que se inicia hasta que se adopta el texto definitivo. La visualización por fechas permite seguir el procedimiento, con indicación de todas las intervenciones de las instituciones y organismos participantes en la toma de decisiones.
} 
datos -y su cruce con otras variables de interés - se presentarán los principales resultados del análisis. Por tanto, la variable dependiente es el sentido del voto de los Estados miembros, pudiendo ser "sí", "no" o "abstención". Si bien, se considerará el voto negativo y la abstención bajo una misma categoría frente al voto afirmativo, tal como se ha realizado en otros estudios previos de la literatura (Arregui y Thomson 2014).

El análisis se divide en dos partes. En la primera parte, la variable dependiente es analizada en función de diferentes variables de clasificación ofrecidas por el dataset elaborado. De este modo, existen clasificaciones en función de la configuración del Consejo en el que se ha votado, el área política, el procedimiento legislativo y el tipo de ley. Tanto la configuración del Consejo como el área son dos clasificaciones temáticas. La primera se refiere a la formación del Consejo que se reúne, mientras que la segunda se refiere a la temática exacta de la votación. Se ha decidido incluir ambas para poder profundizar en el análisis, ya que existen 10 formaciones del Consejo, pero hasta 18 áreas políticas. El procedimiento de voto se clasifica en función de si se trata de procedimiento legislativo ordinario (codecisión), consulta y/o assent, que han sido los tres principales procedimientos legislativos en el periodo de tiempo analizado. Finalmente, el tipo de ley distingue según sean reglamentos, directivas y decisiones principalmente, ya que son los tres tipos de ley que son vinculantes para los Estados miembros.

En la segunda parte se estudiará la relación entre la variable dependiente y el voto de los Estados miembros, con otras variables relativas a ciertas características de los distintos países que forman parte del Consejo de Ministros de la UE. Los Estados miembros pueden ser clasificados en función de diversos criterios, como la ideología de sus gobiernos, su tamaño o su dependencia o interrelación económica respecto a otros países de la UE. Estas variables pueden presentar relaciones con el voto, concretamente con la frecuencia con la que un Estado miembro emite un voto negativo. A través de correlaciones bivariadas, se presentarán las posibles relaciones entre estos factores. La existencia de correlación no implica causalidad, si bien para que exista una causalidad es condición previa cierta relación. En esta parte se quiere estudiar estas posibles relaciones con las características propias de los Estados miembros.

El primer factor es el tamaño de un estado, medido como la media de los millones de habitantes que residen para el periodo estudiado, según datos de Eurostat. A mayor población, mayor poder de voto en el Consejo y más capacidad de influir en el proceso de negociación y de formar parte de coaliciones ganadoras. España se sitúa como el quinto país en población y poder formal de voto, por detrás de Alemania, Reino Unido, Francia e Italia.

El segundo factor es la interdependencia económica, medida como la importancia que representan los intercambios comerciales en exportaciones e importaciones para la economía de cada Estado miembro. Estos datos han sido obtenidos a partir de Eurostat y se ha calculado el porcentaje del PIB que representan para cada país y año del periodo estudiado, realizando finalmente una media de las dos legislaturas. Este flujo comercial es asimétrico y bidireccional, es decir, la importancia 
del flujo comercial no es recíproca y un Estado miembro pequeño dependerá mucho más respecto a un Estado miembro grande, que viceversa. Por ejemplo, en el caso de España los países que dependen más de su comercio exterior con ella no son los mismos de los que depende España. Portugal es el país donde mayor porcentaje del PIB representa el comercio con España, con una media del 15,7\% para el periodo estudiado. En cambio, Portugal es el cuarto país para España, con un 2,9\% del PIB, por detrás de Francia, Alemania e Italia.

El tercer factor es la ideología de los gobiernos, medida en una escala de izquierda-derecha. Para ello, se ha recurrido al dataset de Christopher Wratil ${ }^{4}$ (2020), que mide la posición ideológica de todos los gobiernos de la UE hasta el año 2019. Se calcula a partir del cruce de datos de Comparative Manifesto Project (CMP) ${ }^{5}$ con los datos de composición de los gabinetes de ParlGov Project ${ }^{6}$. El CMP calcula la ideología de los partidos a partir de sus programas electorales. El dataset CMP Goverment Positions Data mide cual es la ubicación ideológica de un gabinete en concreto, ponderando en función de la composición del gobierno por diferentes partidos ${ }^{7}$. Se han obtenido los datos para cada año del periodo estudiado y gobierno de la UE mediante la ponderación de los diferentes gabinetes en el poder en función de la cantidad de días que han permanecido en el cargo.

\section{ANÁLISIS DESCRIPTIVO}

Cabe recordar que el Consejo de la UE funciona a partir del consenso como principio (Lewis 2008), donde la unanimidad es una institución informal utilizada en una amplia mayoría de votaciones, incluidas aquellas que se rigen mediante el sistema de mayoría cualificada. En la práctica, un voto en contra y una abstención tienen el mismo efecto, porque en el sistema de mayoría cualificada, por el cual se realizan la mayoría de las votaciones, para que una votación salga adelante requiere el voto a favor del $55 \%$ de los Estados que representen como mínimo el 65 \% de la población de la Unión.

Los datos analizados confirman el funcionamiento de este principio, pues de las 32.784 observaciones realizadas, tan solo 838 son de votos en contra o abstenciones, un 2,56 \% del total. Si se estudia respecto al número de votaciones, el 64,8\% se realizan por unanimidad, en el 19\% se contabiliza alguna abstención ${ }^{8}$ y en el 16,2\% algún voto

\footnotetext{
${ }^{4}$ Wratil, Chrhistopher. 2020. CMP Government Positions Data.

${ }^{5}$ El Comparative Manifesto Project (CMP) o The Manifesto Project (https://manifesto-project.wzb.eu/) analiza el papel de los partidos en diferentes etapas del proceso político y examina específicamente la calidad de la representación programática.

${ }_{6}$ ParlGov (https://www.parlgov.org/) es una infraestructura de datos para la ciencia política, que contiene información para todas las democracias de la UE y la mayoría de la OCDE. La base de datos combina aproximadamente 1.700 partidos, 1.000 elecciones ( 9.400 resultados) y 1.600 gabinetes.

7 Malta queda excluida al no disponer de datos para su estudio. Así como, existen datos perdidos para algunos años de los siguientes países: Polonia, República Checa y Eslovenia.

8 Cabe remarcar que, desde el referéndum del Brexit, el Reino Unido se ha abstenido de forma considerable en un amplio número de votaciones, mientras el resto de los Estados miembros ha votado a favor.
} 
negativo. Por tanto, el número de casos en que los Estados miembros no votan a favor es muy limitado, aún con un número tan amplio de observaciones.

En el Gráfico 1 se observa que los cinco estados que más veces han votado en contra (o se han abstenido) son Reino Unido (15,4\%), Austria (5,3\%), Alemania (4,6\%), Países Bajos $(4,4 \%)$ y Polonia $(4,1 \%)$. El caso del Reino Unido sobresale especialmente, ya que se sitúa a gran distancia del siguiente, pues ya desde el inicio del Brexit se ha abstenido de forma sistemática en la mayoría de las votaciones del Consejo, incrementando todavía más su oposición a las votaciones con un elevado 15,4 \% de votos emitidos que no son a favor. Por otro lado, los países que menos veces votan en contra son Francia (0,3\%), Lituania $(0,3 \%)$, Grecia $(0,8 \%)$ y Chipre $(0,8 \%)$. España se situaría en un punto medio del gráfico, con un $2 \%$ de sus votos en contra o abstenciones, cerca de otros países como Bélgica, Portugal o Eslovaquia.

\section{Gráfico 1: Voto en contra por Estado miembro en el Consejo de la UE (2009-2019)}

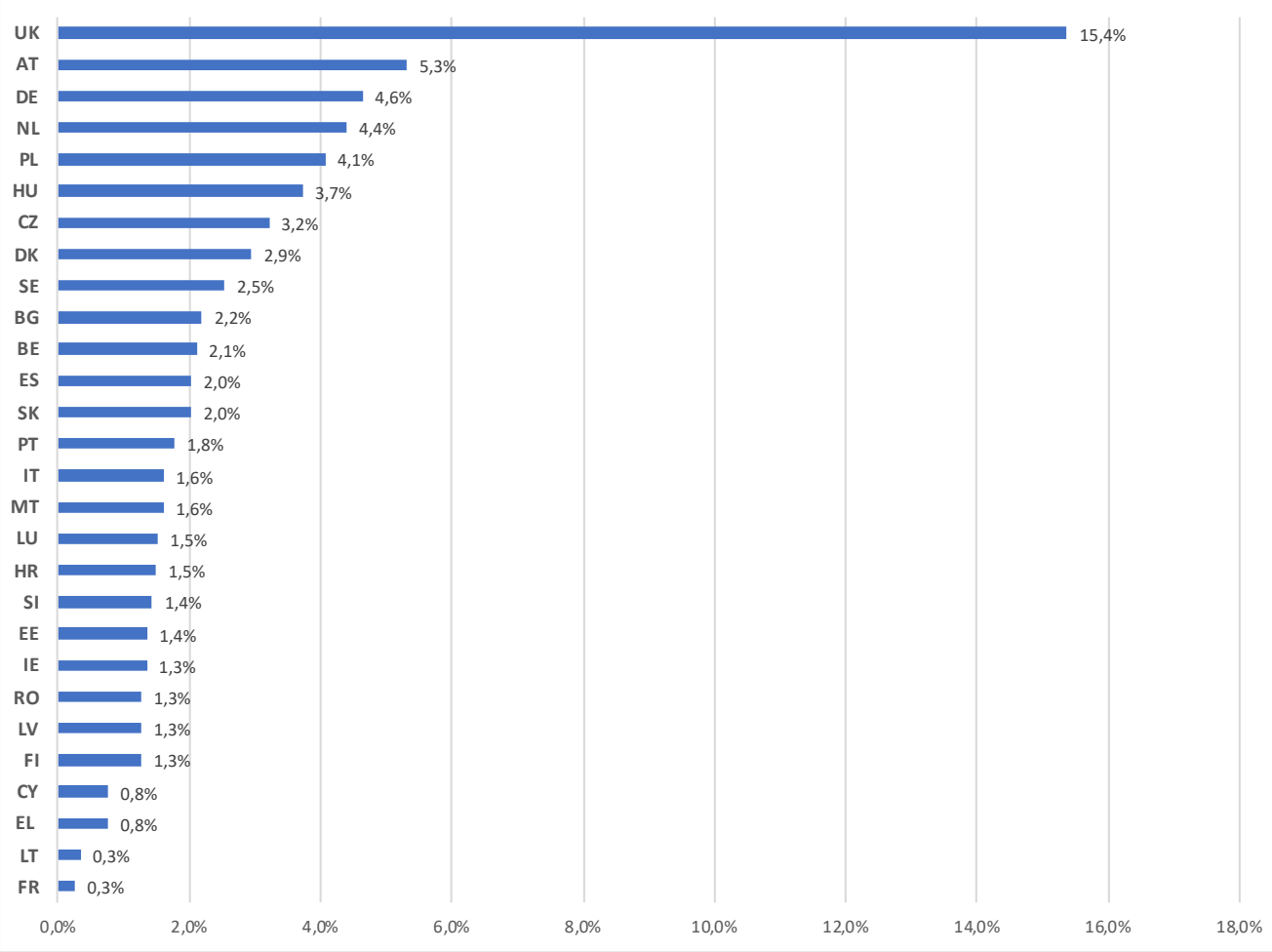

Fuente: Elaboración propia a partir de datos de Votewacth.eu

Los datos permiten señalar algunas tendencias y clasificaciones del voto preliminares. Por ejemplo, algunos de los países denominados aportadores netos al presupuesto de la UE (Reino Unido, Austria, Alemania, Países Bajos o Dinamarca) tienden a votar más en contra que otros históricamente receptores (Grecia, Chipre o Italia). Algunos países del Este (Hungría, Polonia o República Checa) se ubican en el lado de los que más votan en contra, mientras que los Estados bálticos (Estonia, Letonia y Lituania) se ubican en el lado de los que más votan a favor.

Se puede profundizar en el caso español y comparar con otros países de tamaño similar o con características parecidas. Los países de su entorno Mediterráneo y del sur 
(Grecia, Italia y Portugal) tienen unos menores porcentajes de voto en contra, mientras que otros países grandes (Alemania o Polonia) tienen mayores porcentajes de voto en contra. Por tanto, España se sitúa en torno al punto medio, tanto si se compara con la totalidad de la UE, como si la comparación se realiza respecto a países con tamaños de población similares. Sin embargo, es cierto que España es el país del sur que mayor porcentaje de votos en contra presenta, por encima de Italia.

El Gráfico 2 muestra el voto de los 28 Estados miembros y España, respectivamente desagregado, por la composición del Consejo, es decir, en función de la temática y de los ministros designados para representar los intereses de un Estado. Tal y como indica este gráfico, en los 28 Estados miembros todas las configuraciones de Consejo se encuentran con unos porcentajes de voto negativo entre el $2 \%$ y 3,5\%. Las formaciones que cuentan con mayor porcentaje en contra son la de Empleo, Política Social, Salud y Consumo (3,5\%), seguida de la de Educación, Juventud, Cultura y Deporte $(3,4 \%)$ y la de Justicia e Interior $(3,2 \%)$. Por otro lado, las que cuentan con un menor porcentaje de votos en contra son la de Economía y Finanzas (2,0\%), Competitividad $(2,1 \%)$ y Agricultura y Pesca $(2,1 \%)$. El resto de las configuraciones del Consejo se sitúan en valores entre el 2,3\% - 3\% de votos negativos. Los datos indican que aquellas áreas en las que la UE tiene más competencias y capacidad de coordinación (agricultura, competitividad y economía) son las que tienen menos oposición, mientras que aquellas en las que las competencias siguen residiendo fundamentalmente en los Estados y que, por tanto, son más sensibles para estos (interior, política social, empleo y educación) cuentan con mayor oposición.

\section{Gráfico 2: Voto en contra de la UE-28 y España por configuración del Consejo (2009-20 19) ${ }^{9}$}

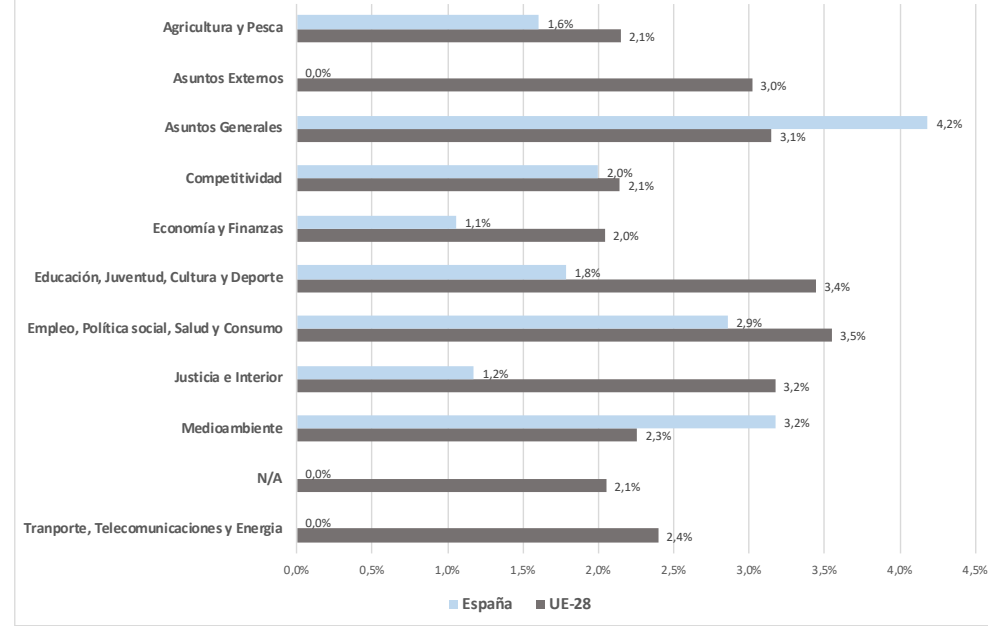

Fuente: Elaboración propia a partir de datos de Votewacth.eu

\footnotetext{
${ }^{9}$ No se ha incluido la categoría "Procedimiento Escrito", este tipo de categoría no es un Consejo en sí mismo sino un procedimiento establecido por el reglamento del Consejo para que los Estados puedan emitir su voto telemáticamente en algunas votaciones muy específicas y de carácter urgente. España cuenta con un $16,7 \%$ de votos en contra ( 5 de 6 votaciones).
} 
Si se miran los datos para el caso de España, los resultados difieren poco. Las formaciones del Consejo de Asuntos Generales (4,2\%), Medio Ambiente (3,2\%); y Empleo, Política social, Salud y Consumo $(2,9 \%)$ son las que cuentan con mayor oposición por parte de España. Por otro lado, en dos configuraciones, España siempre ha votado a favor durante el periodo estudiado: Asuntos Exteriores y Transporte, Telecomunicaciones y Energía. El resto de formaciones, se encuentran con cifras de rechazo menores al $2 \%$.

Si se comparan las cifras con el conjunto de la UE, España acostumbra a votar menos en contra en todas las reuniones del Consejo de manera sistemática, excepto en Asuntos Generales y de Medio Ambiente, que son donde más se ha opuesto, en comparación con el resto de los 27 Estados miembros. En las otras formaciones del Consejo, las cifras de apoyo (en el caso de España) se sitúan por encima de la media.

Este análisis también se puede realizar en función del área política, es decir, la temática de la propuesta que no tiene por qué coincidir necesariamente con la formación del Consejo. Esta categoría ofrece un mayor rango de opciones y por tanto mayor capacidad explicativa para poder profundizar. El Gráfico 3 muestra el voto de los 28 Estados miembros, y España respectivamente desagregado, por el tipo de área política. Las áreas con mayor nivel de rechazo son Control Presupuestario (6,6\%), Asuntos Constitucionales e Interinstitucionales (4,6\%), Agricultura (4,4\%) y Medio Ambiente y Salud Pública (4,4\%). Por otro lado, las áreas con menor contestación son Comercio Internacional (0,8\%), Asuntos Económicos y Monetarios (0,9\%), Desarrollo Regional (1,3\%), Desarrollo (1,4\%) y Pesca $(1,4 \%)$. El resto de áreas se sitúan con valores entre el $2 \%-4 \%$.

Gráfico 3: Voto en contra de los Estados miembros y España por área política (2009-2019)

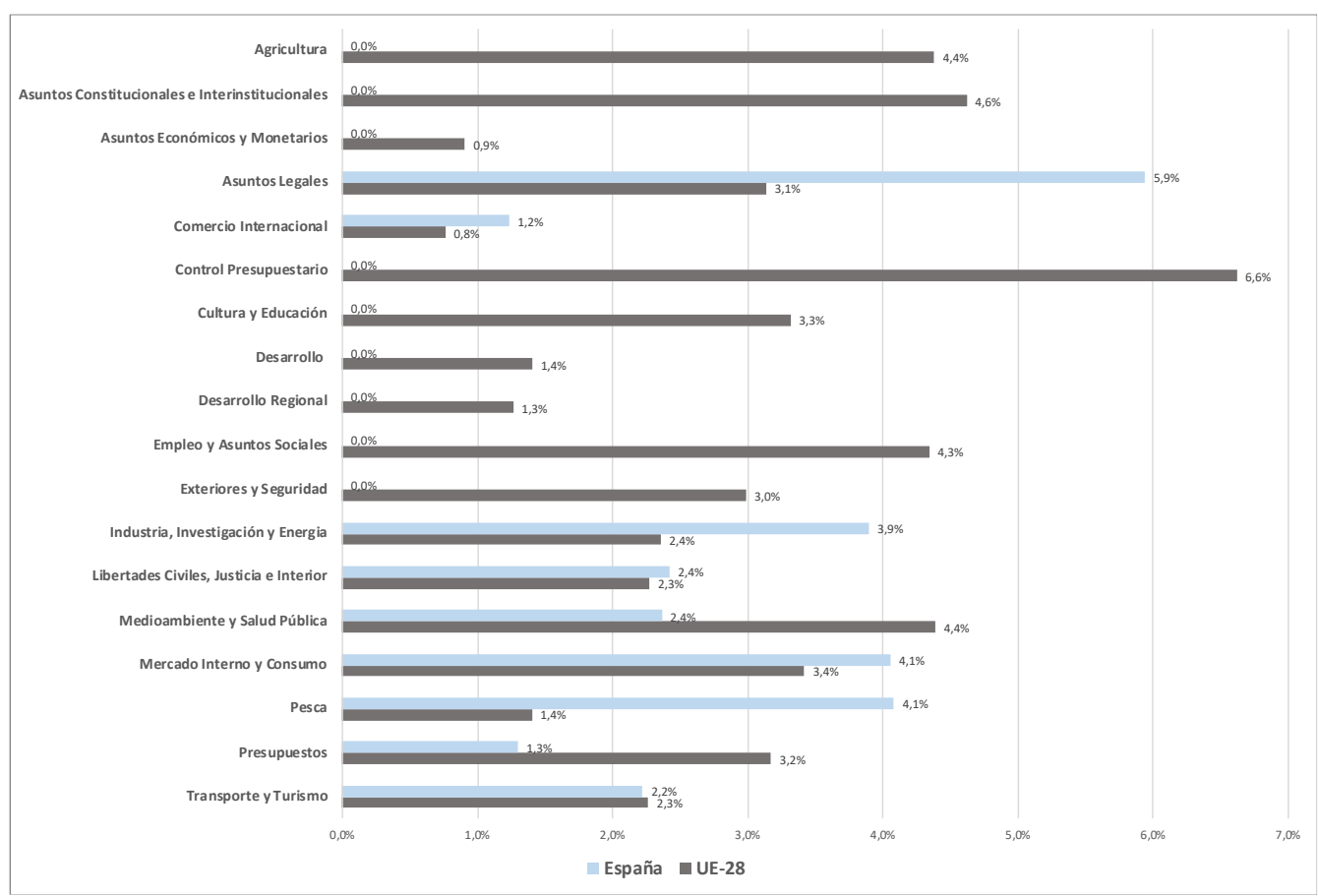

Fuente: Elaboración propia a partir de datos de Votewacth.eu 
Para el caso individual de España, los resultados varían un poco respecto a las áreas con mayor contestación. En este caso, son Asuntos Legales (5,9\%), Mercado Interno y Consumo (4,1\%), Pesca (4,1\%); e Industria, Investigación y Energía (3,9\%) las áreas en las que más ha votado en contra España. Por otro lado, existen 9 áreas en las que España nunca ha votado en contra (Agricultura, Asuntos Constitucionales e Interinstitucionales, Cultura y Educación, Desarrollo, Asuntos Económicos y Monetarios, Empleo y Asuntos Sociales, Exteriores y Seguridad, Desarrollo Regional, Control Presupuestario).

Cabe destacar las diferencias en torno a la temática de "Pesca", ya que para España es un área política clave y una de las más controvertidas, mientras que a nivel europeo es un área con menor oposición que el resto. Sin embargo, el área "Agricultura", la cual concentra una parte muy sustantiva del presupuesto europeo y que también es de especial interés para España, no cuenta con un solo voto en contra en comparación con los 28, que sí se encuentra por encima de la media en cuanto a nivel de oposición se refiere. En el resto de áreas políticas, España vota más a favor que el conjunto de la UE, con unos niveles de rechazo situados por debajo del 4\%, cifra ligeramente inferior a la del resto de los 27 Estados miembros.

Una vez analizadas las votaciones en función de las temáticas y formaciones del Consejo, el Gráfico 4 explica el voto desagregado por tipo modalidad legislativa de los 28 Estados miembros, y España respectivamente. Para los 28, las votaciones relativas a presupuestos $(4,2 \%)$ y directivas $(3,4 \%)$ son las que reciben mayores votos en contra, mientras que las decisiones $(1,6 \%)$ y reglamentos $(2,2 \%)$ las que menos. Cabe recordar que la gran mayoría de legislación aprobada se concentra en directivas y reglamentos. Si se compara con España, en este caso son las directivas $(3,0 \%)$ las que generan un mayor número de votos en contra/abstenciones, mientras que las decisiones tan solo suponen un $0,9 \%$. Siguiendo la tendencia de los anteriores datos, todos los valores se sitúan de media por debajo del total de los 28.

Gráfico 4: Voto en contra de los Estados miembros y España por tipo de ley (2009-2019)

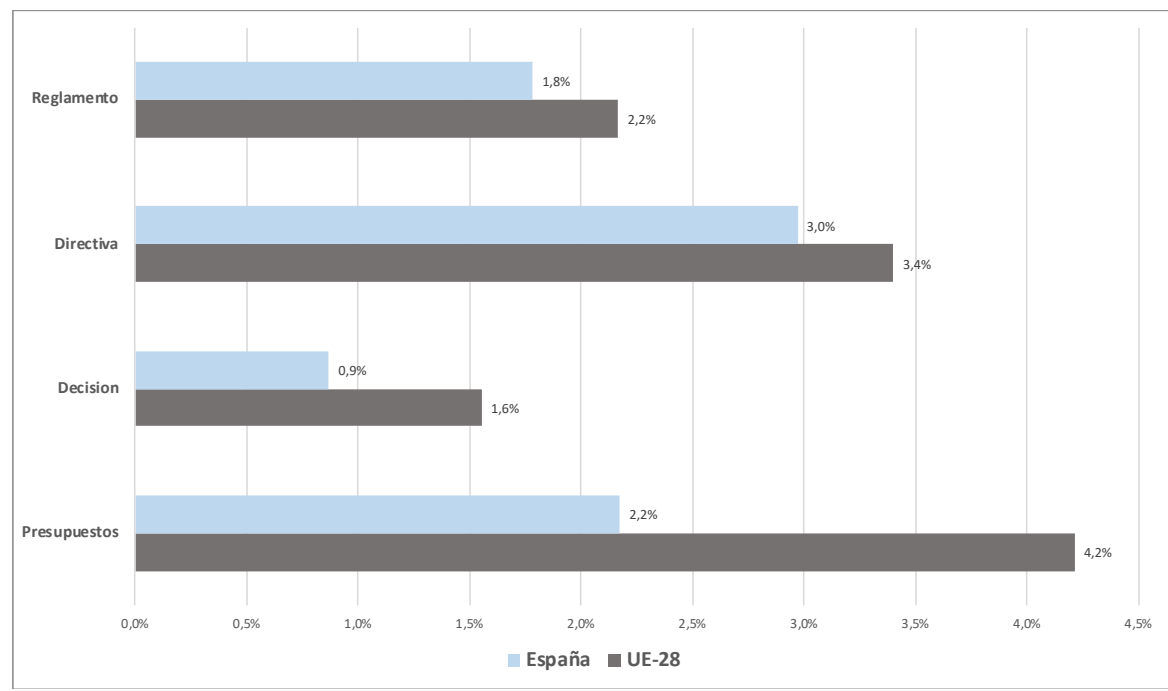

Fuente: Elaboración propia a partir de datos de Votewacth.eu 
Otra manera de clasificar las votaciones es en función del procedimiento, el Gráfico 5 muestra el voto desagregado por tipo de procedimiento. El procedimiento legislativo ordinario (codecisión) es el más utilizado desde la entrada en vigor, en 2009, del Tratado de Lisboa porque es el procedimiento establecido para que tanto el Parlamento Europeo como Consejo participen en la redacción final de las leyes europeas. Este procedimiento se sitúa con un nivel de voto en contra/abstenciones del 2,6\% para los 28 y del 2,2\% para España. Las votaciones que más rechazo generan son las relativas al Fondo de Solidaridad de la UE, sin embargo, representan muy pocos casos en términos absolutos, la siguiente categoría con más votos en contra es Presupuestos $(4,1 \%)$. Por otro lado, el procedimiento de assent nunca ha recibido votos en contra de ningún estado. En el caso español, tres categorías cuentan con un $100 \%$ de votos a favor (Acuerdo, Solicitud, Consulta). Por otro lado, otra vez la votación sobre el Fondo de Solidaridad de la UE (25\%) es la más controvertida, seguida del Procedimiento No Legislativo $(8,3 \%)$ que triplican el valor respecto al conjunto de los Estados miembros.

Gráfico 5: Voto en contra de los 28 Estados miembros y España por tipo de procedimiento (2009-2019)

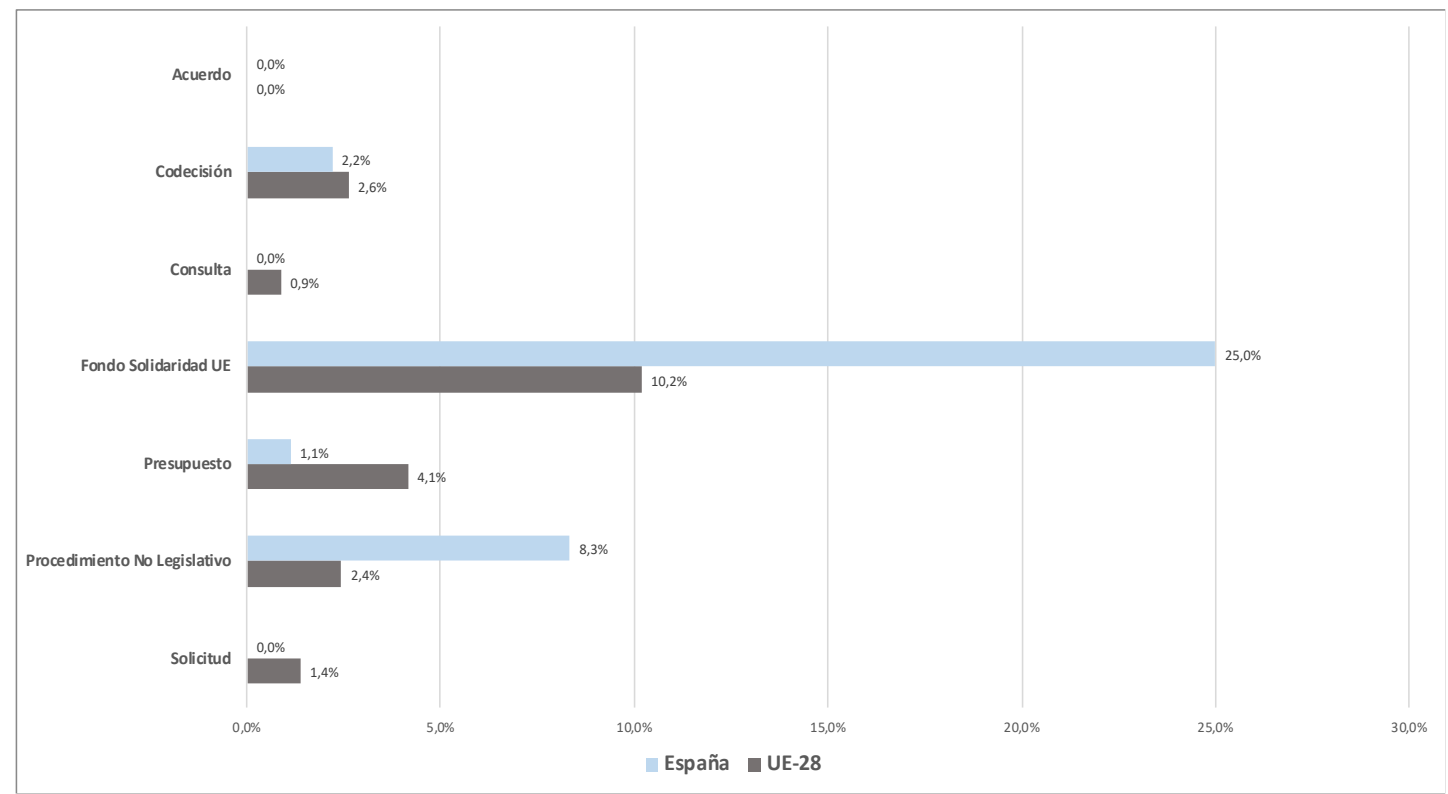

Fuente: Elaboración propia a partir de datos de Votewacth.eu

Tras haber analizado el voto de España y del resto de los Estados miembros, en función de diferentes variables de clasificación, a continuación, se va a profundizar en el voto de España y junto a qué países vota en contra. En total, de 1.185 votaciones estudiadas, tan solo en 24 ocasiones no ha votado a favor, emitiendo 12 votos negativos y 12 abstenciones. El Gráfico 6 muestra qué países han coincidido en no votar a favor cuando España tampoco lo ha hecho. 
Gráfico 6: Estados miembros que han votado con España cuando no ha votado a favor (2009-2019)

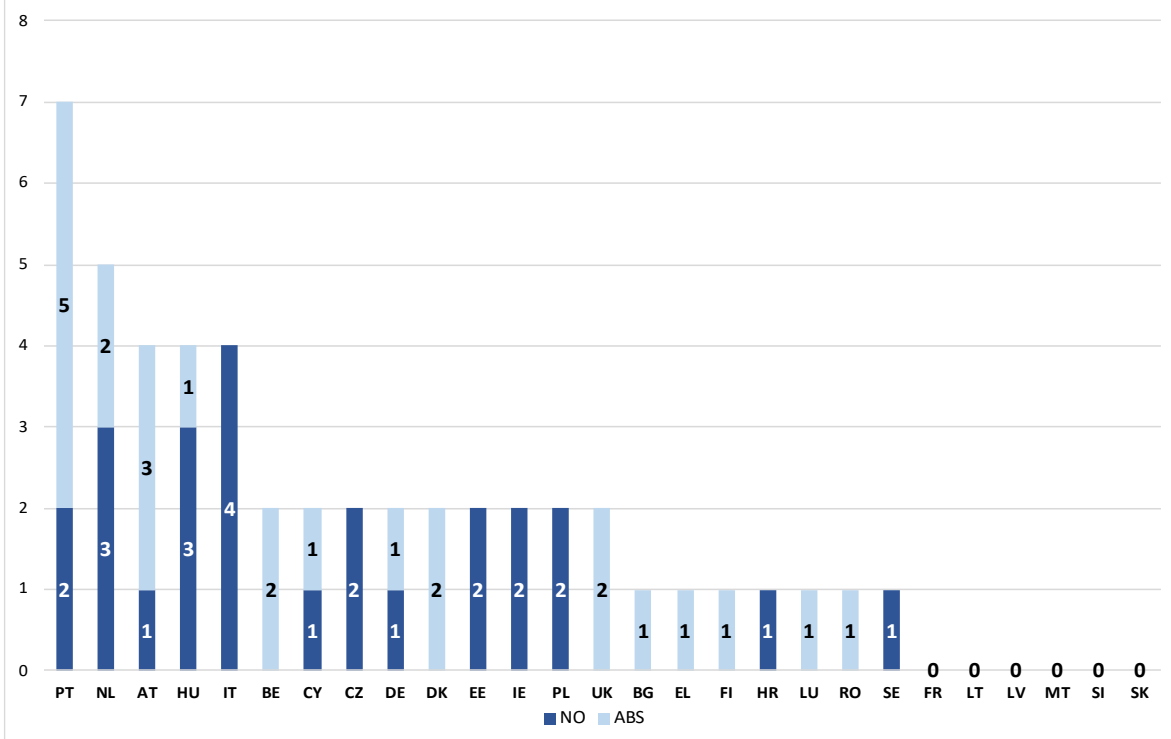

Fuente: Elaboración propia a partir de datos de Votewacth.eu

Portugal con 2 votos en contra y 5 abstenciones es el Estado miembro que más ha votado con España, es decir, en casi un tercio de las ocasiones. Le siguen Países Bajos (3 negativos y 2 abstenciones), Italia (4 negativos), Austria (1 negativo y 3 abstenciones) y Hungría (3 negativos y 1 abstención). El resto de Estados miembros, se sitúan en 2 o 1 votos y, finalmente, un grupo de 6 países (Eslovaquia, Eslovenia, Francia, Letonia, Lituania y Malta) que nunca han votado en contra o abstención con España. Esto es significativo en la medida en que la literatura ha mostrado que existen redes de cooperación entre Estados miembros que pueden influir tanto en el proceso de negociación como de decisión dentro del Consejo de Ministros (Huhe et al. 2021).

El grupo de países que más ha votado con España es diverso y no obedece a un solo criterio. El primero, Portugal, es un Estado miembro que tiene una única frontera y es con España y, por tanto, una mayor dependencia económica, a lo que hay que añadir que ambos forman parte de la red de los países del sur de Europa que, de acuerdo a la literatura, muestran ciertos patrones de cooperación en el Consejo de Ministros (Huhe et al, 2021). El segundo, Países Bajos, destaca por su alta posición en el gráfico y habría que analizar en profundidad en qué votaciones se han producido estas coincidencias para explicar esta relación. El tercero es Italia y comparten la ubicación geográfica y ser considerados uno de los estados grandes en población de la UE. Al igual que con Portugal, España e Italia comparten redes de cooperación en distintos ámbitos (Huhe et al. 2017; Huhe et al. 2021). Finalmente, los dos siguientes, Austria y Hungría, están situados en un área geográfica diferente y con menos dependencia económica y características en común que con otros posibles países del entorno. 
A continuación, presentamos la Tabla 1 que cruza los cinco Estados miembros que más han votado en contra con España y ofrece información sobre en qué configuraciones del Consejo se han producido estas coincidencias.

Tabla 1: Estados miembros y configuraciones del Consejo en las que más han votado en contra con España (2009-2019)

\begin{tabular}{|l|l|}
\hline Estado Miembro & Configuración del Consejo \\
\hline Portugal & $\begin{array}{l}\text { Asuntos Generales (3); Empleo, Política Social, Salud y Consumo (2); } \\
\text { Educación, Juventud, Cultura y Deporte (1), Justicia e Interior (1) }\end{array}$ \\
\hline Países Bajos & $\begin{array}{l}\text { Agricultura y Pesca (1); Asuntos Generales (1); Competitividad (1); } \\
\text { Procedimiento Escrito (1); Educación, Juventud, Cultura y Deporte (1) }\end{array}$ \\
\hline Italia & $\begin{array}{l}\text { Asuntos Generales (1); Competitividad (1); Economía y Finanzas (1); } \\
\text { Educación, Juventud, Cultura y Deporte (1). }\end{array}$ \\
\hline Austria & Agricultura y Pesca (2); Asuntos Generales (1), Medio Ambiente (1) \\
\hline Hungría & $\begin{array}{l}\text { Agricultura y Pesca (2); Asuntos Generales (1); Empleo, Política Social, } \\
\text { Salud y Consumo (1). }\end{array}$ \\
\hline
\end{tabular}

A nivel general, el Consejo de Asuntos Generales es el que más frecuencia presenta, seguido de Agricultura y Pesca, tal como se observó en el análisis específico de esta variable. España ha votado en contra. con Portugal, en 3 de las 7 ocasiones en el Consejo de Asuntos Generales, sin embargo, no se observa ninguna votación en otras áreas en las que comparten intereses comunes, como podría ser Agricultura y Pesca. Con los Países Bajos no se observa ninguna concentración, ni tendencia de votos en contra en ningún Consejo específico, sino que están repartidos a lo largo de las diferentes formaciones del Consejo; al igual que con Italia, donde destaca que coinciden en una ocasión en el ECOFIN ${ }^{10}$. Finalmente, con Austria y Hungría presenta dos coincidencias a destacar, en Agricultura y Pesca y en Asuntos Generales.

\section{ANÁLISIS: CORRELACIONES Y GRÁFICOS DE DISPERSIÓN}

La frecuencia con la que los Estados miembros votan en contra puede estar correlacionada con diferentes factores que ayudan a explicar el fenómeno. Correlación no implica causalidad, pero para que exista causalidad es necesaria cierta correlación. A continuación, se van a estudiar las relaciones entre el porcentaje de votos en contra/abstenciones con algunos factores demográficos, ideológicos y económicos que puedan ayudar a explicar la variabilidad del voto de los Estados miembros.

En primer lugar, el Gráfico 7 muestra la correlación con la población. La cantidad de habitantes es una variable que sirve para estudiar el tamaño de un país y

\footnotetext{
${ }^{10}$ Consejo en su formación de Asuntos Económicos y Financieros (ECOFIN).

https://www.consilium.europa.eu/es/council-eu/configurations/ecofin/
} 
su posición en el Consejo, ya que a mayor población mayor peso relativo tiene su voto. En el gráfico se aprecia una correlación positiva moderada de coeficiente $r$ de 0,44 entre ambas variables. A mayor población, y por tanto mayor peso en el Consejo, más oposición. Aun así, hay que tener en cuenta la existencia de casos atípicos que se alejan de la tendencia, como Reino Unido o Francia, dos países con una población similar compitiendo por el segundo puesto, pero siendo los países que más y menos votan en contra.

Gráfico 7: Correlación entre población y \% de voto en contra/abstención (2009-2019)

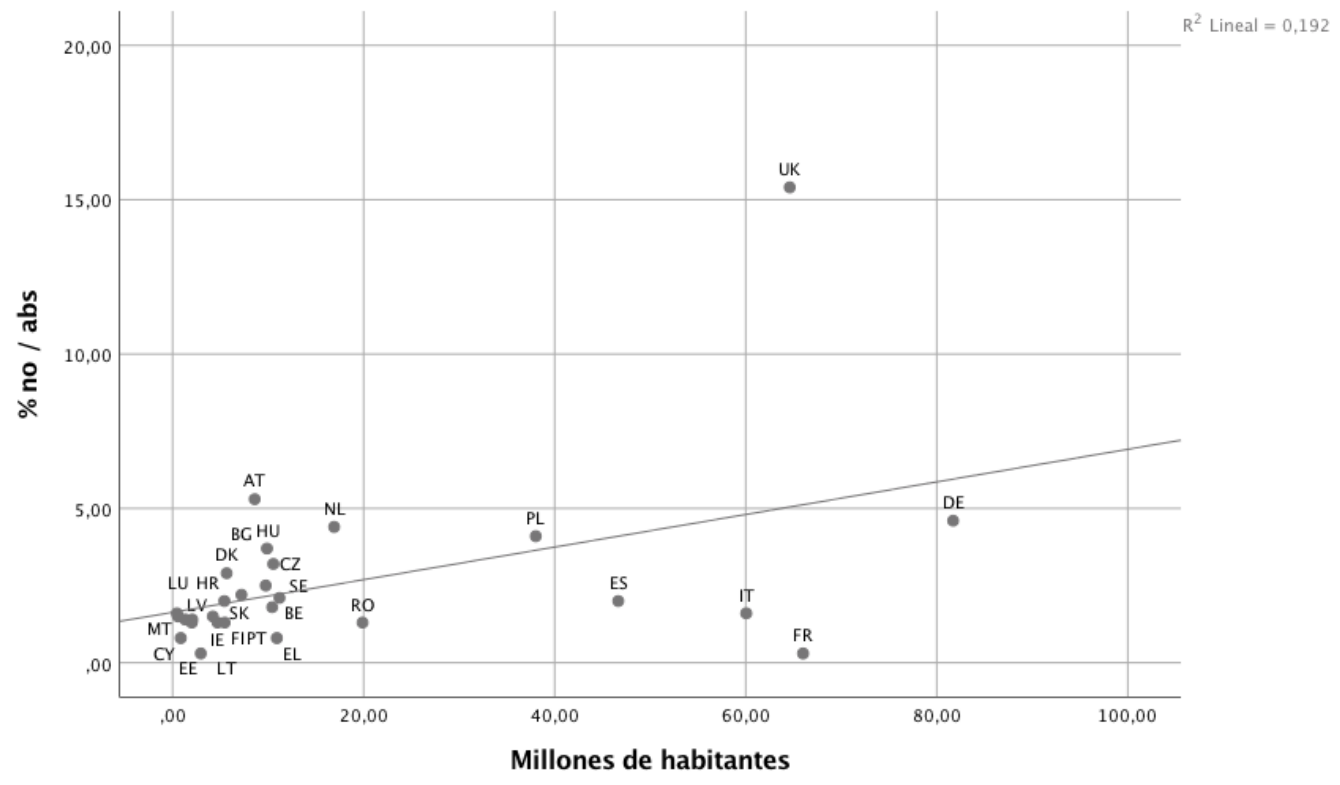

Fuente: Elaboración propia

A continuación, el Gráfico 8 muestra la correlación con la ideología. La ubicación ideológica está medida como la media de posición en la escala izquierda-derecha de los gobiernos en el periodo analizado. Unos valores más negativos indican posiciones más a la izquierda y unos valores más positivos más hacia la derecha. La relación entre ambas variables es positiva y moderada, inferior a la anteriormente analizada con una $r$ de 0,35. Es decir, los países con gobiernos escorados más hacia la derecha se oponen más en las votaciones, mientras que los países con gobiernos más hacia la izquierda votan más a favor. España se sitúa en el lado de los gobiernos más ubicados a la izquierda, concretamente en el quinto lugar con una media de -12,06, ocupando Francia la posición de gobiernos más a la izquierda y Hungría más a la derecha para el periodo analizado de 10 años. 


\section{Gráfico 8: Correlación entre ideología y \% de voto en contra/abstención (2009-2019)}

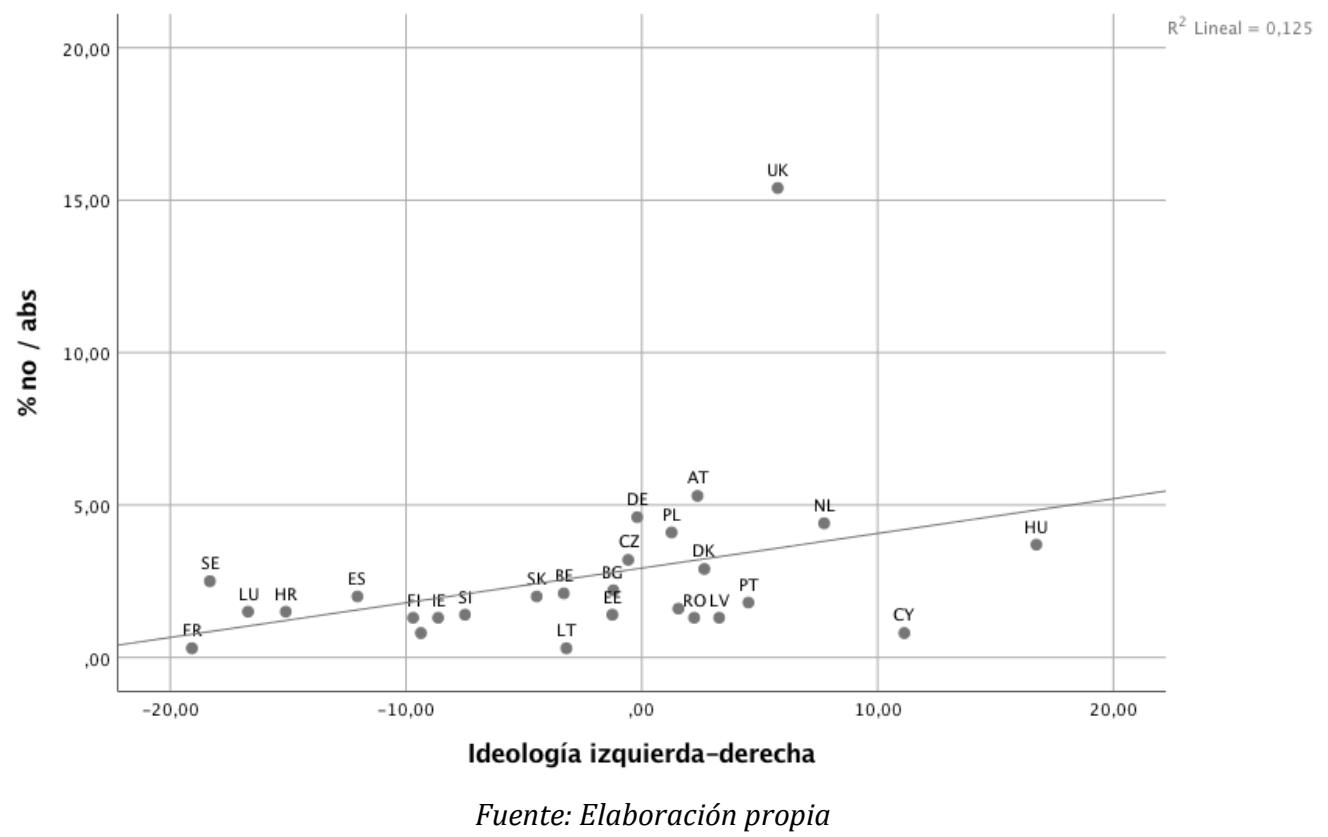

En tercer lugar, se analiza la relación con la dependencia económica, concretamente con el porcentaje del PIB que representa la suma de comercio exterior de cada país con el conjunto de los Estados miembros. Sin embargo, no se ha encontrado una correlación clara entre ambas variables, pues su coeficiente es muy reducido, tan solo de un - 0,12 . En cualquier caso, es negativo y, como se esperaba, a mayor dependencia económica del resto de la UE, menos porcentaje de votos en contra. El país que menos dependencia económica tiene es Grecia, junto a los Estados miembros más grandes (Reino Unido, Italia, España y Francia). Sin embargo, Alemania no se sitúa en este grupo, ya que el comercio intra-UE representa un mayor porcentaje de su PIB. Por otro lado, Eslovaquia, República Checa, Bélgica y Hungría cuentan con altas tasas de dependencia económica respecto a sus vecinos de la UE.

Uno de los motivos por los que no se ha encontrado esta relación clara entre mayor dependencia económica del bloque comunitario y porcentaje de votos en contra es que las relaciones económicas entre los Estados miembros son asimétricas y bidireccionales. Por esta razón, es mejor, posiblemente, analizar estas relaciones bidireccionales: estudiar la correlación entre la dependencia económica hacia un Estado miembro en concreto y la frecuencia con la que votan en contra, formando coaliciones. De acuerdo con esta lógica, se ha procedido a analizar un caso concreto, tal como presenta el Gráfico 9, que muestra los resultados del cruce entre dependencia económica hacia España y las veces que han votado juntos, en sentido negativo, para cada Estado miembro. 
Gráfico 9: Correlación entre dependencia económica respecto a España y $\%$ de voto en contra/abstención (2009-2019)

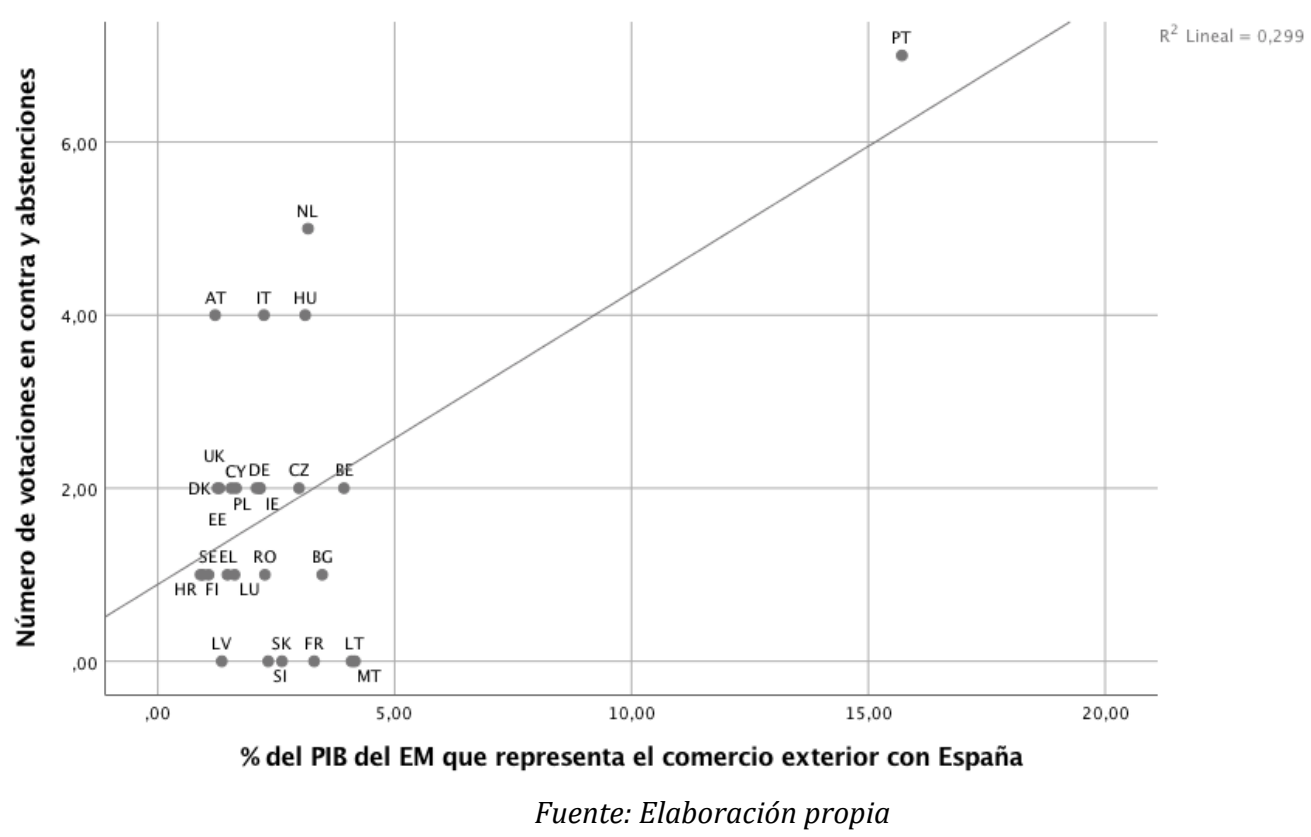

En este análisis los resultados sí muestran un alto nivel de correlación, el más alto de los anteriores, con una $r$ de 0,54. A mayor dependencia, mayor es el número de votaciones en las que los Estados miembros han votado con España. El caso de Portugal es el más destacado, pues el comercio con España representa más de un 15,7\% de su PIB, pero tan solo un 2,9\% para España, más de cinco veces menos. Portugal ha votado hasta en 7 ocasiones - de 24- en sentido negativo junto a España. Países Bajos es otro caso destacado, siendo el segundo país que más veces ha coincido en votar en contra con España (5) y situándose también en la franja de mayores dependencias económicas. Sin embargo, en este caso hay que tener en cuenta la menor asimetría que existe con Portugal. Para Países Bajos, el comercio con España representa el 3,17\% de su economía, pero para España es el 2,1\%, unas relaciones más simétricas que en el caso portugués. Por tanto, para el caso español se puede establecer cierta correlación entre la formación de coaliciones opositoras y la dependencia económica.

Si se miran las relaciones de dependencia económica desde la perspectiva contraria, es decir, introduciendo como variable la dependencia económica que tiene España respecto otros Estados miembros, los resultados cambian. En este caso, el coeficiente de correlación es más bajo situándose en 0,26. Si bien es cierto que establece cierta tendencia positiva entre dependencia económica de España con otros Estados miembros y frecuencia con la que votan en contra, esta relación es menor que la mostrada con los datos anteriores. Por ejemplo, Francia que es el país de cuyo comercio más depende España, situándose en torno a un $6 \%$ del PIB, nunca ha coincidido votando en contra con España. Alemania, que representa el 5,7\%, ha votado dos veces en contra; mientras que Portugal, que representa la mitad que Alemania $(2,9 \%)$, lo ha hecho en 7 ocasiones. Por tanto, se demuestra la asimetría de relaciones 
económicas y su impacto en el voto de los Estados miembros. España, al ser considerado uno de los grandes Estados miembros de la UE, tiene mayor influencia en el PIB del resto de Estados miembros, especialmente los pequeños —que viceversay así se refleja en los datos analizados.

\section{CONCLUSIÓN}

El análisis realizado en este artículo es el primer análisis que muestra, de un modo sistemático, datos sobre el comportamiento de voto de España y otros Estados miembros en el Consejo de Ministros de la UE en las dos últimas legislaturas (20092019). Además, identifica una serie de posibles variables explicativas del comportamiento del voto de los Estados miembros.

Los resultados empíricos han mostrado que la proporción de votos en contra o abstenciones ha sido muy limitada por parte de los Estados miembros, con la excepción del Reino Unido y, en menor medida, de Austria y Alemania. El caso de España se situaría en una posición media, con tan sólo en un $2 \%$ de todas las votaciones llevadas a cabo dentro del Consejo de Ministros de la UE en el periodo analizado. Este hallazgo está en líneas generales con los patrones encontrados en estudios previos (Mattila 2004, 2008, 2009; Hayes-Renshaw et al. 2006; Hagemann 2008; Hosli et al. 2011; Plechanová 2011). Por tanto, muchos Estados miembros, incluida España, votan a favor incluso en aquellos temas que no están de acuerdo con la decisión final adoptada. Esta es una institución informal ya internalizada por parte de los Estados miembros (Lewis 2008). Por tanto, en el comportamiento de voto de los Estados miembros tenemos dos mecanismos que están operando. Por un lado, existe una amplia cultura de consenso en el Consejo de Ministros de la UE, que hace que votar en contra tenga más costes que beneficios para los Estados miembros en el término medio (ver Lewis 2008). Por otro lado, una posible explicación del mecanismo que está aquí operando para explicar los pocos casos de disenso en el Consejo de Ministros. Puede ser que cuando los Estados miembros precisan enviar un mensaje o una señal de oposición (a algunos actores domésticos) sobre una decisión adoptada, es cuando tiene lugar el disenso. Por ejemplo, el hecho de que en un país existan intereses domésticos activos muy bien organizados incrementa la probabilidad de que los Estados miembros muestren disenso en el voto final del Consejo (Arregui y Thomson 2014). Esta lógica también explicaría por qué el Reino Unido ha sido el Estado miembro que más veces ha votado en contra en el periodo de tiempo analizado. Reino Unido tenía una opinión pública y unos actores domésticos que habían apostado públicamente por el Brexit durante el periodo de tiempo analizado, de ahí que votar en contra en el Consejo de Ministros podía tener un efecto positivo para el gobierno británico, dada la creciente coalición de actores pro-Brexit y el activismo de los medios de comunicación británicos con lo que sucede en el día a día de Bruselas.

Los resultados del análisis llevado a cabo muestran, también, que algunos de los países denominados contribuyentes netos al presupuesto de la UE tienden a votar más 
en contra que los países receptores. También se evidencia que los países con gobiernos escorados hacia la derecha se oponen con más frecuencia en las votaciones que los países con gobiernos de izquierda. El análisis también indica que cuanto más grande es un Estado miembro, es más probable que vote en contra o que se abstenga en el Consejo. Esto tiene que ver, posiblemente, con el hecho de que los Estados miembros más grandes tienen que defender una diversidad de intereses mayor y todos los temas que se deciden en Bruselas tienen consecuencias domésticas importantes, mientras que para los Estados miembros más pequeños hay temas que no son tan relevantes o, sencillamente, son irrelevantes.

Con relación al caso de España, muestra un mayor grado de disenso que otros países del sur de Europa (Grecia, Italia o Portugal), mientras que un menor grado de disenso que otros países grandes de la UE (Alemania o Polonia). Por otro lado, las áreas temáticas en las que España muestra más disenso son Medio Ambiente, Empleo y Salud, mientras que, en otras áreas como Política Exterior o Políticas de Transporte y Energía, España ha mostrado un consenso total con el resto de Estados miembros en el periodo de tiempo analizado. Finalmente, la investigación también muestra evidencia empírica de que cuanto mayor es la dependencia económica de España con otros Estados miembros, mayor es la formación de coaliciones opositoras en las votaciones del Consejo. Este hallazgo puede explicarse de acuerdo con la Teoría Integubernamental de Moravcsik (1998). Según Moravcsik, el comportamiento de un Estado miembro en el proceso de decisión está vinculado con los patrones de interdependencia significativos que existen entre los Estados miembros. En el caso de España, esta investigación confirma dicho argumento, sobre todo, en lo que se refiere a la interdependencia económica de España con otros Estados miembros.

Para concluir, el consenso es claramente una institución que pervive en el Consejo a lo largo del tiempo, incluso en periodos de intensa crisis económica. De hecho, esta investigación ha mostrado que los países que han sufrido en mayor medida la crisis económica de los últimos años (fundamentalmente los países del sur de Europa) no han cambiado sus patrones de disenso con relación a periodos anteriores. En estudios futuros habría que incluir qué papel desempeñan tanto el Parlamento Europeo como la Comisión a la hora de explicar este tipo de patrones. Además, en el futuro también sería interesante examinar las condiciones a partir de las cuales determinados factores explicativos son más importantes que otros. 


\section{FINANCIACIÓN}

Este trabajo ha contado con el apoyo del programa Erasmus+ de la Unión Europea, en el marco de los Proyectos 611941 - EPP- 1-2019-1-ES-EPP-JMO- CoE (Barcelona Center for European Studies ${ }^{11}$ ) y 600488 - EPP- 1- 2019- 1- ES - EPPJMO- Chair (Jean Monnet Chair in EU Governance ${ }^{12}$ ).

\section{REFERENCIAS}

Achen, C. (2006). Institutional realism and bargaining models. In R. Thomson et al. (Eds). The European Union Decides (p. 86-123). Cambridge University Press. https://doi.org/10.1017/CB09780511492082.005

Arregui, J. (2012). Problemas de Legitimidad democrática, representación y rendimiento de cuentas en el proceso político de la Unión Europea. Cuadernos Europeos de Deusto, 46, 85-112. https://doi.org/10.18543/ced-46-2012pp85112

Arregui, J. (2015). Relaciones económicas UE-EEUU: negociación e implicaciones del TTIP. Revista CIDOB d'Afers Internacionals, 110, 43-66.

https://www.cidob.org/ca/articulos/revista_cidob_d_afers_internacionals/11 0/relaciones_economicas_ue_eeuu_negociacion_e_implicaciones_del_ttip

Arregui, J. \& Thomson, R. (2014). Domestic adjustment costs, interdependence and dissent in the Council of the European Union. European Journal of Political Research, 53(4), 692-708. http://dx.doi.org/10.1111/1475-6765.12060

Arregui, J. \& Creighton, C. (2018). Public Opinion and the Shaping of Immigration Policy in the European Council of Ministers. Journal of Common Market Studies, 56(6), 1323-1344. https://doi.org/10.1111/jcms.12745

De Mesquita, B. B. (1994). Political forecasting: An expected utility method. In B. B. de Mesquita \& F. N. Stokman (Eds.). European community decision-making: models, applications and comparisons, p. 71-104. Yale University Press. http://www.jstor.org/stable/j.ctt1dszx1f

\footnotetext{
11 https://www.upf.edu/web/baces

$12 \mathrm{https}: / / w w w . u p f . e d u / w e b / e u-g o v e r n a n c e$
} 
Hagemann, S (2008). Voting, statements and coalition-building in the Council from 1999 to 2006. In D. Naurin \& H. Wallace (Eds). Unveiling the Council of the European Union: Games governments play in Brussels, p. 36-63. Palgrave Macmillan. https://doi.org/10.1057/9780230583788_3

Hayes-Rensahw, F. \& Wallace, H. (2006). The Council of Ministers (2nd Edition). Palgrave Macmillan.

Hayes-Renshaw, F., Van Aken, W. \& Wallace, H. (2006). When and why the EU Council of Ministers votes explicitly. Journal of Common Market Studies, 44 (1), 161-194. https://doi.org/10.1111/j.1468-5965.2006.00618.x

Hosli M.O., Mattila M. \& Uriot M.C.J. (2011), Voting in the Council of the European Union after the 2004. Enlargement: A Comparison of Old and New Member States. Journal of Common Market Studies, 49(6), 1249-1270. https://doi.org/10.1111/j.1468-5965.2011.02196.x

Høyland, B., \& Hansen, V. W. (2014). Issue-specific policy-positions and voting in the Council. European Union Politics, 15(1), 59-81. https://doi.org/10.1177/1465116513495970

Huhe, N., Thomson, R., Arregui, J. \& Naurin, D. (2021). Intergovernmental cooperation, networks, national policy positions and partisan ideologies: longitudinal evidence from the Council of the EU. Journal of European Public Policy, 29 (1), 78-96. https://doi.org/10.1080/13501763.2021.1991980

Lewis, J (2008). Strategic bargaining, norms and deliberation. In D. Naurin \& H. Wallace (Eds), Unveiling the Council of the European Union: Games governments play in Brussels, p. 165-184. Palgrave Macmillan. https://doi.org/10.1057/9780230583788_9

Mattilla, M. (2004). Contested decisions: Empirical analysis of voting in the European Union Council of Ministers. European Journal of Political Research, 43 (1), 29-50. https://doi.org/10.1111/j.1475-6765.2004.00144.x

Mattilla, M. (2008). Voting and coalitions in the Council after the enlargement. In D. Naurin \& H. Wallace (Eds). Unveiling the Council of the European Union: Games governments play in Brussels, p. 23-35. Palgrave Macmillan. https://doi.org/10.1057/9780230583788_2 
Mattila, M. (2009). Roll call analysis of voting in the European Union Council of Ministers after the 2004 enlargement. European Journal of Political Research, 48 (6), 840-857. https://doi.org/10.1111/j.1475-6765.2009.01850.x

Moravcsik, Andrew (1998). The Choice for Europe: Social Purpose and State Power from Messina to Maastricht. Cornell University Press.

Plechanovová, B (2011). The EU Council enlarged: North-South-East or coreperiphery?. European Union Politics, 12 (1), 87-106. https://doi.org/10.1177/1465116510390720

Wratil, Christopher (2020). CMP Government Positions Data. Version 2.0. https://wratil.eu/files/CMP\%20Government\%20Positions\%20Data\%20Versi on\%202.0.pdf 